\title{
Perturbations of Weakly Resonant Power System Electromechanical Modes
}

\author{
Ian Dobson, Senior Member, and Emilio Barocio, Member
}

\begin{abstract}
It is not uncommon for oscillatory electric power system modes to move close to a resonance in which eigenvalues coincide. In a weak resonance, the modes are decoupled and the eigenvalues do not interact. We analyze general perturbations of a weak resonance and find two distinct behaviors, including interactions near strong resonances in which the eigenvalues quickly change direction. The possible perturbations are illustrated with interactions between electromechanical modes in a 4-generator power system. Some of the interactions are similar to subsynchronous resonance and can lead to oscillatory instability.
\end{abstract}

Index Terms-Eigenvalues and eigenfunctions, Hopf bifurcation, oscillations, power system dynamic stability, resonance, root loci, subsynchronous resonance.

\section{INTRODUCTION}

A POWER system mode is an eigenvalue of the system linearization and its associated eigenvector. Since the modes determine the power system stability and small signal transient performance, understanding the behavior of the modes is fundamental to avoiding power system oscillations and ensuring that transients are sufficiently well damped [1]-[3]. Indeed, a better understanding and suppression of low frequency oscillations could relax some of the constraints on larger bulk power transfers and increase power system security.

An exact coincidence of eigenvalues in both frequency and damping is called a resonance. As parameters vary, the power system modes vary and it is not uncommon for two complex eigenvalues to pass near resonance. A basic question is: do the modes interact near resonance? That is, do the eigenvalues move independently or do they interact in some way? This question has been investigated for some time, including work by Van Ness [4], [5] and Klein and Rogers et al. [6] in the late 1970s and early 1980s and more recent work by Kwatny [7], [8], Dobson [9], [10], Auvray and Dobson [11], Jones and Andersson [12] and Nomikos and Vournas [13]. Practical examples are the interactions between several $0.7 \mathrm{~Hz}$ modes in the Western Area of North America [6], [14], [12] and a modal interaction in a

Manuscript received May 21, 2004. This work was supported in part by the National Science Foundation under Grant ECS-9988574 and by CONACYT Mexico under Project 31840-A. This paper was presented in part at the 2003 IEEE Bologna PowerTech Conference, Bologna, Italy, June 2003. Paper no. TPWRS-00061-2004.

I. Dobson is with the Electrical and Computer Engineering Department, University of Wisconsin, Madison, WI 53706 USA (e-mail: dobson@engr. wisc.edu).

E. Barocio was with the Electrical and Computer Engineering Department, University of Wisconsin, Madison, WI 53706 USA. He is now with the Postgraduate Mechanical and Electrical Engineering School, Autonomous University of Nuevo León, Nuevo León, Mexico (e-mail: ebarocio@gama. fime.uanl.mx).

Digital Object Identifier 10.1109/TPWRS.2004.841242 30-generator model of the mid and western areas of Japan [15]. For a detailed review up to 2000, see [9]. Earlier investigators sometimes observed modal interactions, but the effects seemed anomalous, especially when considered from the point of view of diagonalizable linearizations.

There are two types of resonance: strong and weak [16], [9]. Strong resonance has a nondiagonalizable linearization and weak resonance has a diagonalizable linearization. If the pair of eigenvalues is resonant at $\lambda$, the corresponding Jordan blocks of the Jordan canonical form are

$$
\text { strong: }\left(\begin{array}{ll}
\lambda & 1 \\
0 & \lambda
\end{array}\right) \text { and weak: }\left(\begin{array}{cc}
\lambda & 0 \\
0 & \lambda
\end{array}\right) \text {. }
$$

Strong resonance is associated with eigenvalue interaction. In particular, eigenvalues passing near strong resonance sharply change direction. For example, two eigenvalues approaching each other in frequency can turn through a right angle so that they are diverging in damping. Indeed, one of the eigenvalues can become unstable as a consequence of passing near the strong resonance [9]. On the other hand, eigenvalues passing through weak resonance show no interaction. Although strong resonance is more generic than weak resonance, weak resonance can arise due to special features of the power system model. For example, if two areas of the power system are decoupled, then if a local mode in one area is resonant with a local mode in the other area, this will be a weak resonance. Another example is that some (perhaps idealized) power system models may have bilateral symmetry and then pairs of modes arising from the symmetry will have a weak resonance. These issues are further discussed in [9].

In this paper, we analyze eigenvalue movements (root loci) as a parameter is varied. In Section II, we first consider eigenvalue movements that pass exactly through a weak resonance in the complex plane and then perturb this ideal situation to reveal and analyze the two types of eigenvalue movements that can occur. In Section III, the eigenvalue movements are illustrated with near resonant electromechanical modes in a 4-bus power system model. The eigenvalues can interact and change their directions of movement. This modal interaction can lead to oscillatory instability and is the same modal interaction that occurs in subsynchronous resonance. Section IV briefly considers corresponding ideas in mechanics and Section V concludes the paper.

Nonlinear modal interactions are also of interest in power systems [17]-[20], but should be distinguished from the linear (first order) modal interactions considered in this paper. The eigenvalue movements or root loci in this paper are nonlinear curves, but they arise in the usual way from a linearized power system model that changes as a parameter is varied. In applications of the methods of this paper, the parameter varied could arise from 
loadings, controller settings, or uncertain model data such as load parameters, or could smoothly interpolate discrete changes such as loss of a line.

\section{Perturbation of Weak Resonance}

This section summarizes mathematical results that are more carefully stated and proved in the Appendix. The power system differential equations are assumed to have real parameters $t$ and $\epsilon$. The parameter $t$ parameterizes the eigenvalue movement in the complex plane; that is, $t$ is the root locus parameter. The parameter $\epsilon$ controls the perturbation. The two complex eigenvalues of interest are $\lambda_{1}$ and $\lambda_{2}$ and these vary as functions $\lambda_{1}(t, \epsilon)$ and $\lambda_{2}(t, \epsilon)$ of $t$ and $\epsilon$.

Suppose that when $\epsilon=0$ and as $t$ is varied the power system encounters a weak resonance at $t=0$ so that $\lambda_{1}(0,0)=\lambda_{2}(0,0)$. We examine a general perturbation of this situation by letting $\epsilon$ change from zero.

The eigenvalues do not vary smoothly near weak resonance. Therefore, to analyze the eigenvalue movement, we study the smooth functions

$$
\begin{aligned}
& \mu(t, \epsilon)=\left(\lambda_{1}(t, \epsilon)-\lambda_{2}(t, \epsilon)\right)^{2} / 4 \\
& \lambda(t, \epsilon)=\left(\lambda_{1}(t, \epsilon)+\lambda_{2}(t, \epsilon)\right) / 2 .
\end{aligned}
$$

$\lambda$ and $\mu$ determine the eigenvalues to be

$$
\lambda_{1}=\lambda+\sqrt{\mu} \text { and } \lambda_{2}=\lambda-\sqrt{\mu}
$$

where $\lambda$ is the average eigenvalue position and $\mu$ determines the separation of the eigenvalues in both magnitude and angle. That is, $\mu$ determines the relative position of the eigenvalues. The distance between the eigenvalues is

$$
\left|\lambda_{1}-\lambda_{2}\right|=2 \sqrt{|\mu|} \text {. }
$$

In particular, the condition for coincident and resonant eigenvalues is $\mu=0$. Moreover, the relative direction of the eigenvalues is

$$
\angle\left(\lambda_{1}-\lambda_{2}\right)=\frac{1}{2} \angle \mu \text {. }
$$

The main result, obtained in the Appendix by a Taylor series expansion, is that to second order in $t$ and $\epsilon$

$$
\mu(t, \epsilon)=A t^{2}+2 H t \epsilon+B \epsilon^{2}
$$

where $A, B$, and $H$ are complex constants. Equation (6) describes the movement of $\mu$ as $t$ varies as a parabola in the complex plane. When $\epsilon=0$, the parabola is degenerate and lies in a ray from the origin as shown in Fig. 1. When $\epsilon$ is nonzero, the parabola changes as shown in Fig. 1; changing $\epsilon$ has only the effect of magnifying or changing the scale of the parabola. There are two cases: A type A perturbation has the origin outside the parabola and a type $B$ perturbation has the origin inside the parabola. Now we continue to make use of the second order approximation (6) and discuss the effect of these possibilities on the relative eigenvalue movement.

1) Weak resonance with $\epsilon=0$. In this case, (6) becomes $\mu(t, 0)=A t^{2}$. As $t$ varies, $\mu(t, 0)$ moves along a ray through the origin, first toward the origin, then touching the origin at $t=0$, and then away from the origin. This implies that the eigenvalues $\lambda_{1}$ and $\lambda_{2}$ move together in the same direction, coincide in the weak resonance when $\mu=0$, and
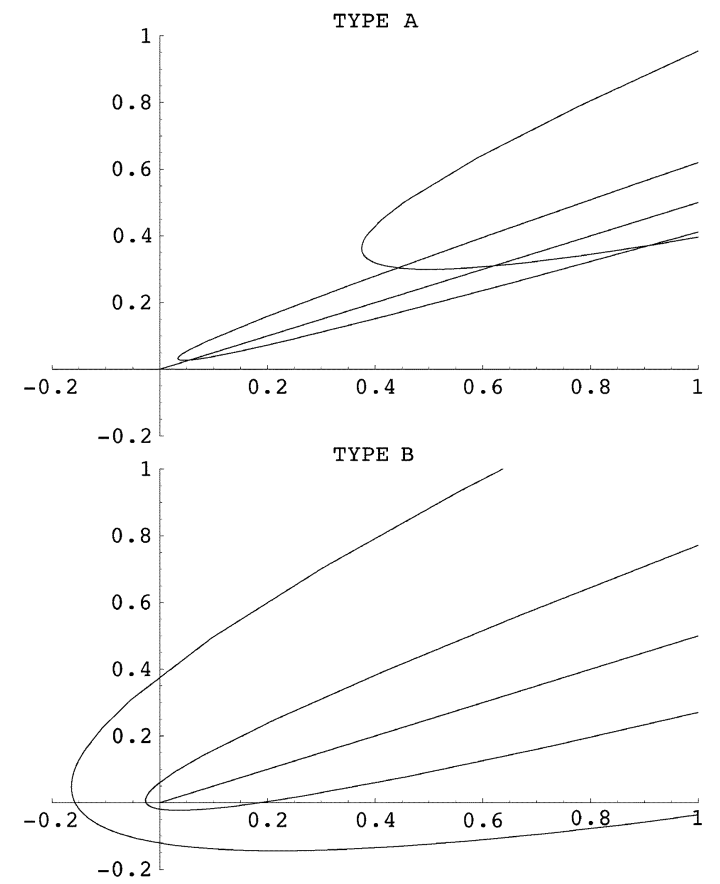

Fig. 1. Example loci of $\mu(t, \epsilon)$ as $t$ varies for $\epsilon=0$ (degenerate parabola in the form of a ray through the origin) and two nonzero values of $\epsilon$ (parabolas of increasing scale as $\epsilon$ increases). Type A has origin outside the parabola and Type B has origin inside the parabola.

then move apart along the same direction. For example, if $\lambda_{1}$ is fixed, then $\lambda_{2}$ moves on a straight line passing through $\lambda_{1}$. There is no interaction in this weak resonance case.

2) Perturbation type A with the origin outside the parabola. As $t$ varies, $\mu(t, \epsilon)$ moves along a parabola toward the origin and then away from the origin. $\mu(t, \epsilon)$ is bounded away from the origin so that the eigenvalues are always separated by a minimum positive distance. If $\epsilon$ is small, then the parabola lies close to the $\epsilon=0$ ray in a bounded region and the eigenvalues move together and separate approximately in the same direction.

3) Perturbation type $B$ with the origin inside the parabola. As $t$ varies, $\mu(t, \epsilon)$ moves along a parabola around the origin. If $\epsilon$ is small, then the parabola lies close to the $\epsilon=0$ ray in some bounded region so that initially and finally the eigenvalues approach each other and separate along approximately the same direction. Near $t=0, \mu$ moves around the origin. Overall, $\angle \mu$ moves through nearly $360^{\circ}$. According to (5), this implies that the eigenvalue relative direction $\angle\left(\lambda_{1}-\lambda_{2}\right)$ moves through nearly $180^{\circ}$. This further implies that there is a $t$ for which $\angle\left(\lambda_{1}-\lambda_{2}\right)$ is at $90^{\circ}$ to the original direction of relative motion. Thus the eigenvalues initially approach each other in a given direction but must move so that at one point they are at right angles to this initial direction.

\section{RESUlTS FOR A 4-GeNERATOR POWER SYSTEM}

Fig. 2 shows a 4-generator power system with two areas based on [6]. Each generator is represented by the same classical model with $H=6.5$ p.u. and damping $D=1.0$ p.u. The per unit line reactances are shown in Fig. 2 and the line 


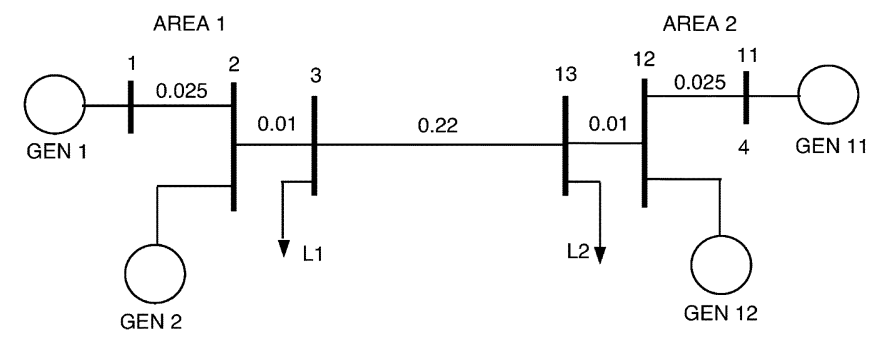

Fig. 2. 4-generator system.
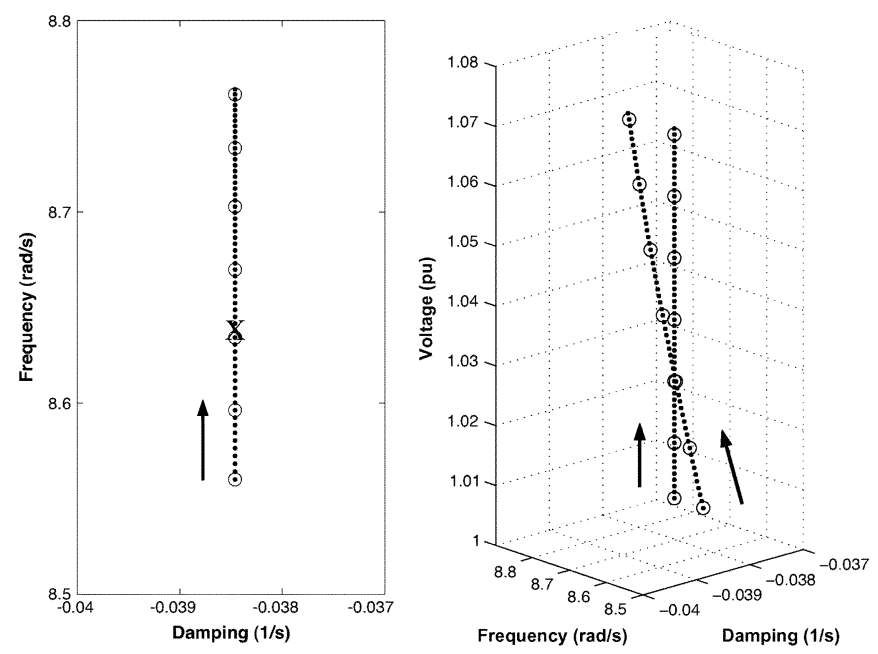

Fig. 3. Eigenvalues passing through weak resonance as bus 2 voltage varies. The $\mathrm{X}$ is the fixed area- 2 eigenvalue and the circles indicate every tenth point.

resistances and line chargings are 0.1 and 1.75 times the line reactances respectively. Generators 1 and 11 each produce 7 p.u. real power and the slack is equally distributed between generators 2 and 12. Each of the loads L1 and L2 are 13.7 p.u. real power and -2 p.u. reactive power from capacitors.

There is an inter-area mode with a frequency of about $0.47 \mathrm{~Hz}$ and two local modes, one in each area, with a frequency of about $1.36 \mathrm{~Hz}$. We study the resonant interaction of the two local modes as their eigenvalues nearly coincide in frequency and damping. In every case, we vary the voltage on bus 2 from 1.0 to 1.07 in order to pass near the resonance. Thus, the bus 2 voltage corresponds to the parameter $t$ considered above.

The weak resonance case is produced by increasing the impedance of the tie line joining the two areas to a very large value (220 p.u.) so that the two areas are effectively decoupled. The area-2 local mode eigenvalue is fixed at the $\mathrm{X}$ in the left half of Fig. 3. The area-1 eigenvalue increases in frequency as bus 2 voltage is increased and passes through the area- 2 eigenvalue in a weak resonance, as shown in Fig. 3.

Restoring the tie line impedance to its base case value (0.22 p.u.) couples the two areas and perturbs the eigenvalue movement to the Type A example shown in Fig. 4. The tie line flow remains zero. Initially, the higher frequency eigenvalue is approximately fixed and the lower frequency eigenvalue moves vertically upward toward the higher frequency eigenvalue. Then the lower frequency eigenvalue becomes approximately fixed and the higher frequency eigenvalue moves vertically upward. In effect, the moving eigenvalue transfers its movement to the fixed eigenvalue when they become close.
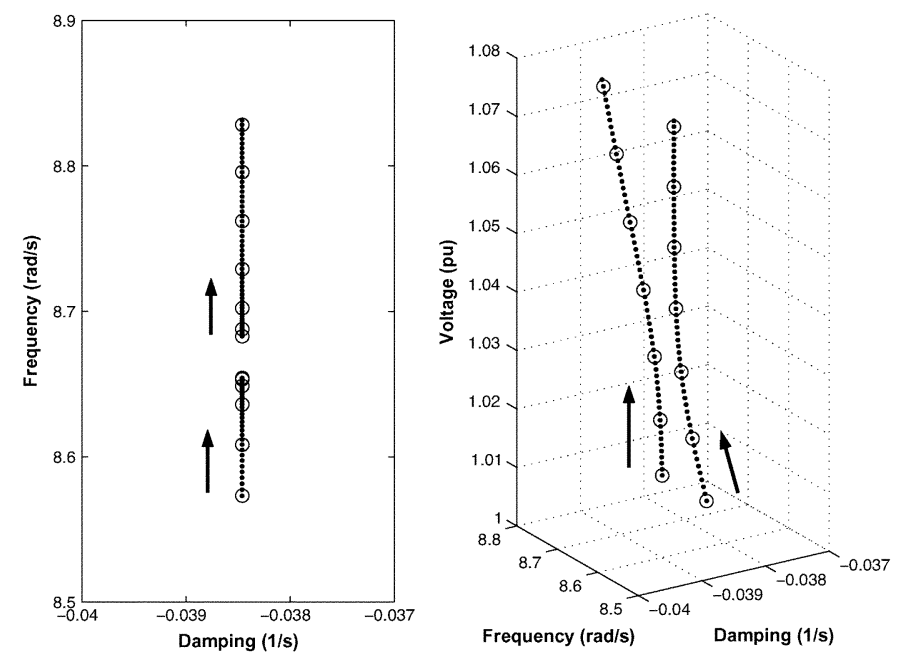

Fig. 4. Type A eigenvalues passing near weak resonance.
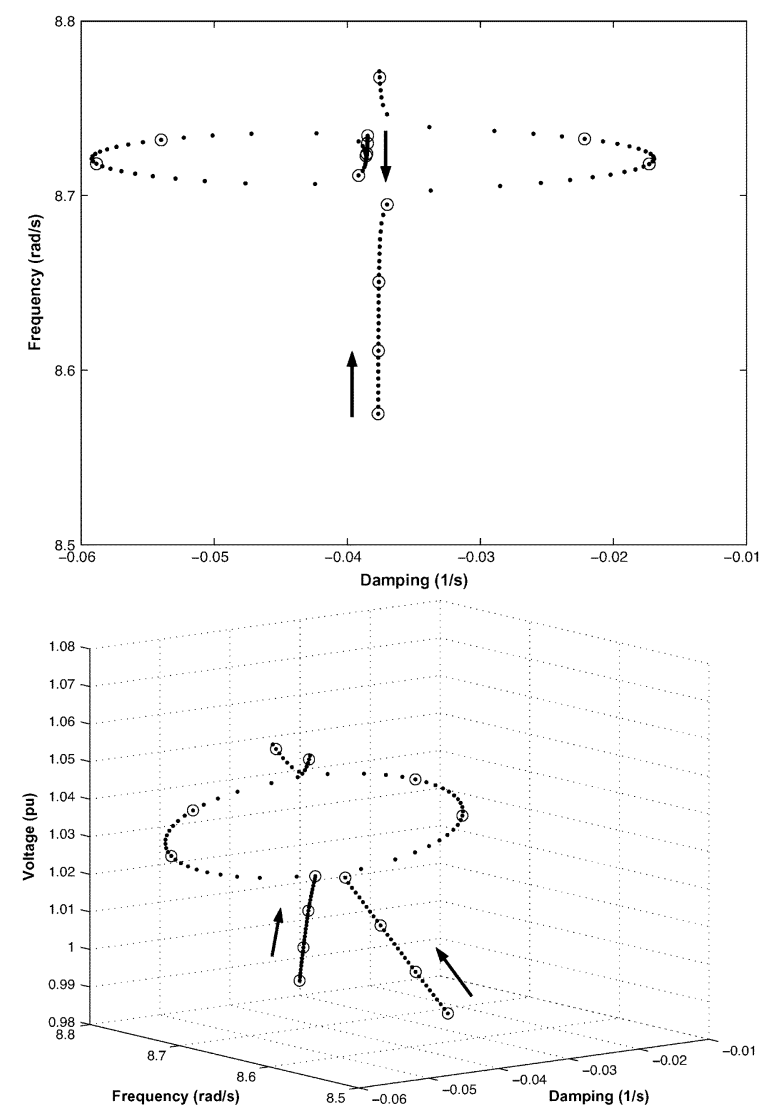

Fig. 5. Type B eigenvalues passing near weak resonance: $D_{1}=D_{2}=0.98$.

A different perturbation of the weak resonance is the type B example shown in Fig. 5. This perturbation is obtained by changing the flows by replacing the distributed slack by a slack at bus 12 and transferring 4 p.u. real power from Load 1 to Load 2. The tie line flow becomes approximately 3.98 p.u. The damping of generators in area 1 is also reduced slightly to 0.98 . These details are summarized in Table I. In Fig. 5, the area-1 eigenvalue moves in a closed curve and the area- 2 eigenvalue generally increases in frequency. The two eigenvalues interact strongly. Their relative direction passes nearly through $180^{\circ}$ 
TABLE I

CAses Simulated (All Data in p.u.)

\begin{tabular}{cccc}
\hline Figs. & L1 & L2 & Changes to base case \\
\hline 3,8 & 13.7 & 13.7 & tie line reactance $=220$ \\
4,9 & 13.7 & 13.7 & \\
5,10 & 9.7 & 17.7 & $D_{1}, D_{2}=0.98$; slack bus 12 \\
6 & 9.7 & 17.7 & $D_{1}, D_{2}=0.6$; slack bus 12 \\
7,11 & 9.7 & 17.7 & $D_{1}, D_{2}=0.1$; slack bus 12 \\
\hline
\end{tabular}

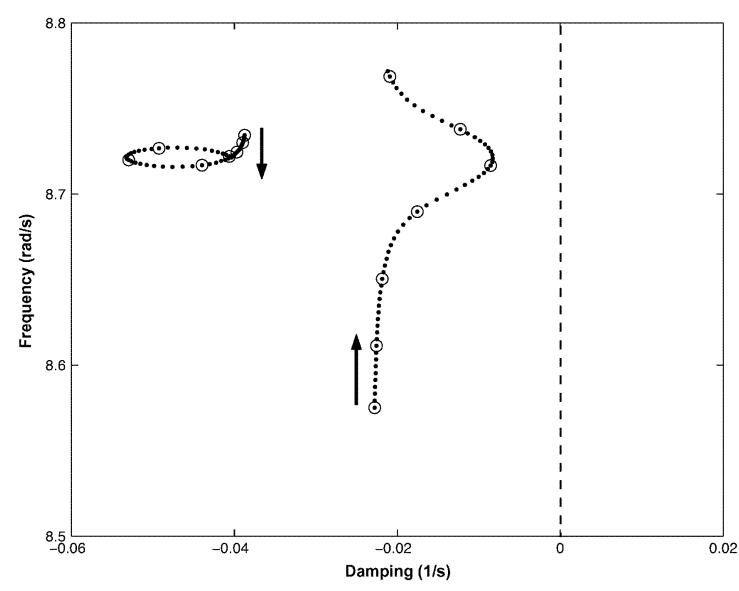

Fig. 6. Medium perturbation type $\mathrm{B}$ eigenvalues passing near weak resonance: $D_{1}=D_{2}=0.6$.

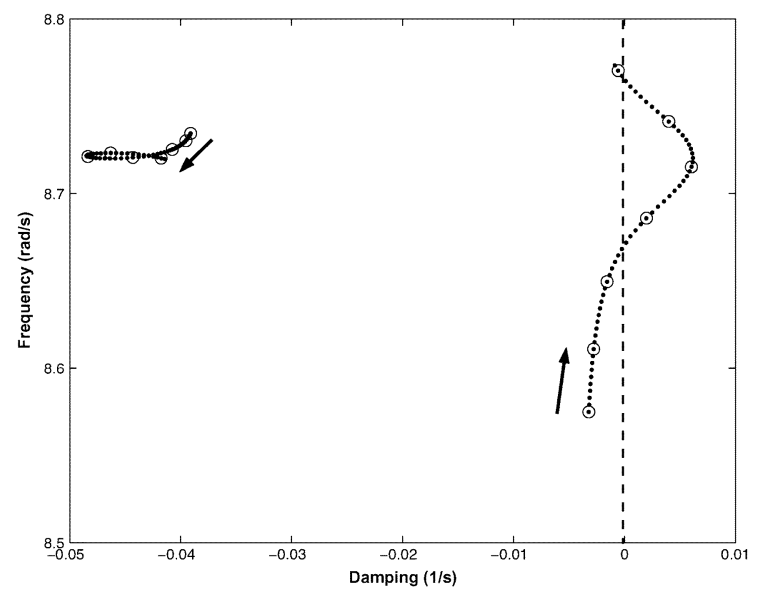

Fig. 7. Large perturbation type B eigenvalues passing near weak resonance: $D_{1}=D_{2}=0.1$.

and the two sharp changes of direction correspond to $\mu$ passing close to the origin and proximity to two strong resonances.

The type B interaction in Fig. 5 is small in amplitude because the perturbation from weak resonance is small. The small amplitude interactions in Fig. 5 could be missed in sampling the modal behavior and in any case have little effect on the system performance. A medium size perturbation from the weak resonance can be obtained by decreasing the damping of the area- 1 generators to 0.6 p.u. and the resulting eigenvalue movement is shown in Fig. 6. A larger perturbation from the weak resonance can be obtained by further decreasing the damping of the area- 1 generators to 0.1 p.u. and the resulting eigenvalue movement is shown in Fig. 7. Now the effect of the interaction is to destabilize one of the electromechanical modes, leading to unstable oscillations.

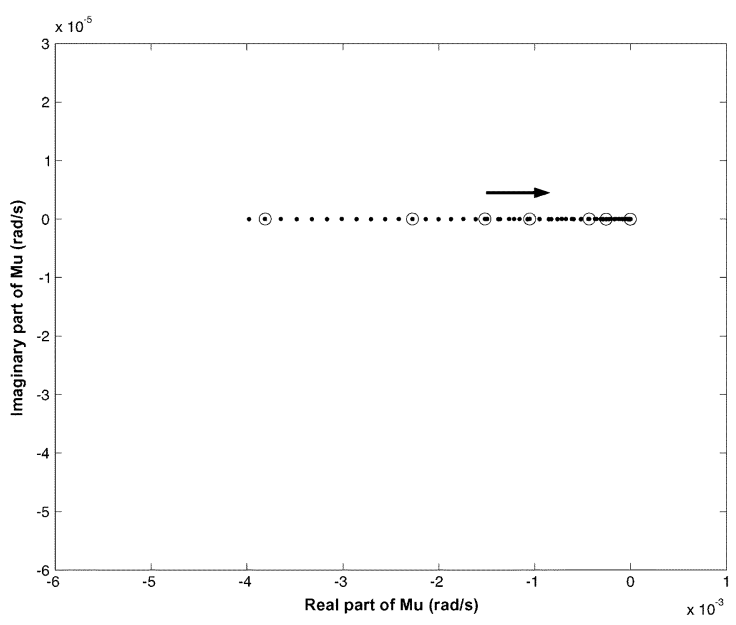

Fig. 8. $\quad \mu$ locus; weak resonance as in Fig. 3.

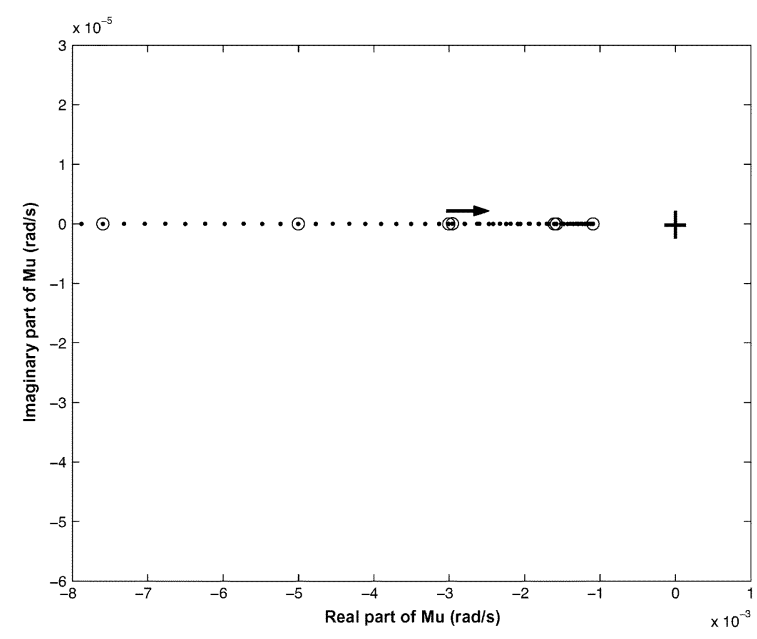

Fig. 9. $\quad \mu$ locus; type A perturbation near weak resonance as in Fig. 4.

We now examine the movement of $\mu$ for some of the cases considered. In the weak resonance case of Fig. 8, the movement of $\mu$ is confined to the negative real axis; starting from a negative value, $\mu$ increases, slows down and touches the origin and then decreases. In the type A perturbation case of Fig. 9, the movement of $\mu$ is similarly confined to the negative real axis, the only difference is that the locus is shifted in a negative direction so that $\mu$ never reaches the origin. For both Figs. 8 and 9 , the movement of $\mu$ is a degenerate parabola confined to the negative real axis because the corresponding eigenvalue movement is vertical. In the type B perturbation case, the parabolic movement of $\mu$ is shown in Fig. 10; note that the vertical scale is one hundred times smaller than the horizontal scale so that the parabola is very close to the real axis. When the real part of $\mu$ is negative and not near zero, its angle is very nearly $180^{\circ}$ and the eigenvalues approach each other vertically. When $\mu$ is real and positive, its angle is $0^{\circ}$ and the eigenvalues are horizontally separated; that is, the eigenvalues have the same frequency. When $\mu$ passes near zero, the angle of $\mu$ and the direction of movement of the eigenvalues changes rapidly. $\mu$ passes very close to zero twice and each passage near zero is a close proximity to a strong resonance corresponding to a sharp change in direction of the eigenvalues of approximately $90^{\circ}$. In the larger type B 


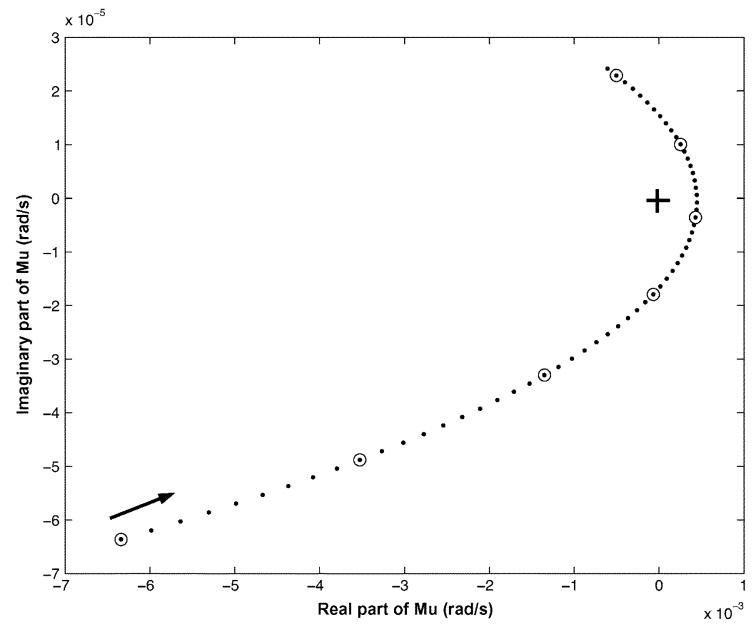

Fig. 10. $\mu$ locus; type B perturbation near weak resonance as in Fig. 5.

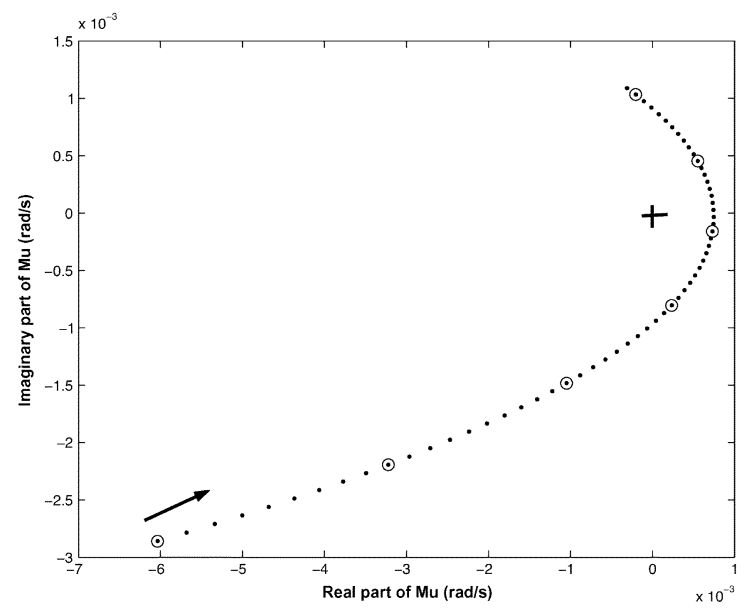

Fig. 11. $\quad \mu$ locus; large perturbation type B as in Fig. 7.

perturbation case of Fig. 11, the movement of $\mu$ has the same parabolic form as Fig. 10; the main difference is that the vertical scale is $10^{-3}$ instead of $10^{-5}$ (the parabola is two orders of magnitude wider).

\section{RElation to Work IN Mechanics}

This section mentions some related work in mechanics. Seyranian [16] has derived formulas for multiparameter perturbations of eigenvalues from weak and strong resonances and shown the geometry of single parameter eigenvalue movements through weak and strong resonances. The eigenvalues do not interact when passing through a weak resonance and turn through a right angle when passing through a strong resonance. These results are illustrated in linear mechanical vibrational problems in which the symmetries of the inertia, damping and restoring force matrices restrict the eigenvalue movements. Of particular interest is the passage through a weak resonance between two real eigenvalues perturbing to passage through two strong resonances that causes a "bubble" of complex eigenvalues between the two strong resonances ([16, Fig. 9]). This perturbation of weakly resonant real eigenvalues corresponds to the type B perturbation of weakly resonant complex eigenvalues considered below. Also interesting is the passage through a weak resonance between two purely imaginary eigenvalues perturbing to eigenvalue movements in which the eigenvalues remain imaginary ([16], Fig. 10). This perturbation of weakly resonant imaginary eigenvalues corresponds to the type A perturbation of weakly resonant complex eigenvalues.

\section{CONCLUSION}

We mathematically analyze generic perturbations of two complex eigenvalues passing through a weak resonance and show that there are two types. The function $\mu$ that describes the relative positions of the eigenvalues traces a parabola or a ray in the complex plane and the two types are distinguished by whether the origin is inside or outside the parabola. In type $\mathrm{A}$, eigenvalues move together and apart without passing each other. In type B, the eigenvalues interact by passing near strong resonances and change their directions of movement. In particular, at one point during the interaction, the relative position of the eigenvalues is at approximately $90^{\circ}$ to its original direction. These analytic results are illustrated by examining eigenvalue movements near resonance of two local area electromechanical modes in a 4-generator power system example. Eigenvalue movement through exact weak resonance is observed when the two areas are effectively decoupled and different perturbations yield the type A and type B eigenvalue movements. Moreover, a larger type B perturbation yields an unstable oscillatory mode caused by the local area mode resonance. It is also possible for a type A perturbation to destabilize eigenvalues initially approaching each other in frequency as shown by two coupled oscillators in [11, Fig. 3].

Consider two complex eigenvalues initially approaching each other in frequency. If the eigenvalues interact as a perturbation of a weak resonance as shown in Fig. 5, the interaction can change their damping, and, if the effect is strong enough, this can lead to oscillatory instability as shown in Fig. 7. The size of the change in damping is proportional to the size of the perturbation. If the eigenvalues interact as a perturbation of a weak resonance as in Fig. 4, then the results of the interaction are much less dramatic because the eigenvalues do not change direction much. However, it is still useful to know that some perturbations of weak resonance are relatively innocuous and what these perturbations look like. Also, if a modal interaction as in Fig. 4 is observed, then the possibility that a modest change in parameters could yield a modal interaction as in Fig. 5 should be considered.

The subsynchronous resonance modal interaction in which series capacitors in transmission lines cause electrical modes to resonate with and destabilize turbine shaft modes is a well known effect [21]-[24]. It has recently been observed that subsynchronous resonance is a perturbation of a weak resonance in which a pair of strong resonances arises [10]. Moreover, Figs. 5, 6, 7 have the same forms as [10, Figs. 9 and 10], which show eigenvalue interactions for the IEEE first benchmark model for subsynchronous resonance. Indeed the subsynchronous resonance eigenvalue interaction is a perturbation of a weak resonance. Viewing both subsynchronous resonance and some electromechanical modal interactions as type B perturbations 
of a weak resonance helps to unify the study of power system oscillations and may help in developing connections to the broader study of oscillations in science and engineering.

Previous work [9] suggested that inter-area oscillations could be caused by two complex power system modes passing near a strong resonance and interacting to cause changes in damping such that one of the modes became oscillatory unstable. In this paper, we suggest another explanation for unstable oscillations in which the interactions are a perturbation of a weak resonance similar to Fig. 5. The weak resonance perturbation explanation is consistent with the explanation in [9] because in each explanation the instability is caused by two modes passing near a strong resonance. However, the structure of the weak resonance perturbation in Fig. 5 is more elaborate because the eigenvalues pass near a second strong resonance to restore stability. That is, a type B perturbation of a weak resonance involves modes passing near a pair of strong resonances. Sensitivity methods to select parameters to minimize the oscillatory instability caused by weak resonance perturbations are explored in [11].

A further step in assessing the importance of these mechanisms for oscillatory instability will require observation of how often they occur in detailed and realistic power system models. One of the contributions of the theoretical analysis in this paper and [9] is that it shows what to look for. The power system example of this paper illustrates in a simple model how electromechanical modes can become unstable via a perturbation of a weak resonance.

\section{APPENDIX}

Assume that the power system differential equations with real parameters $t$ and $\epsilon$ are smooth. The unperturbed system has $\epsilon=0$ and is assumed to have an asymptotically stable equilibrium at $t=0$. Let the Jacobian matrix evaluated at this stable equilibrium be $J(t, \epsilon)$. Then $J$ is a well-defined smooth function of $t$ and $\epsilon$ near $(t, \epsilon)=(0,0)$.

The unperturbed system is assumed to have a weak resonance between two complex modes at $t=0$. In particular, exactly two complex eigenvalues of $J(t, 0)$ coincide at eigenvalue $\lambda$ in a weak resonance ${ }^{1}$ at $t=0$. It follows from the smoothness of the total projection in [25] that the two dimensional complex eigenspace corresponding to both of these two eigenvalues is a smooth function of $t$ and $\epsilon$ near $(t, \epsilon)=(0,0)$. By restricting $J(t, \epsilon)$ to this eigenspace, we obtain a $2 \times 2 \mathrm{com}$ plex matrix $M(t, \epsilon)$ which captures the eigenstructure of the two modes of interest. (That is, if $P(t, \epsilon)$ is the matrix of the total projection onto the two dimensional complex eigenspace and $Q(t, \epsilon)$ is the matrix of the injection of the two dimensional complex eigenspace into the state space, then $M(t, \epsilon)=$ $P(t, \epsilon) J(t, \epsilon) Q(t, \epsilon)$.) The complex matrix $M(t, \epsilon)$ is a smooth function of $t$ and $\epsilon$ near $(t, \epsilon)=(0,0)$. We write

$$
M(t, \epsilon)=\left(\begin{array}{ll}
a(t, \epsilon) & b(t, \epsilon) \\
c(t, \epsilon) & d(t, \epsilon)
\end{array}\right)
$$

\footnotetext{
${ }^{1}$ Two other complex conjugate eigenvalues of $J(t, 0)$ also coincide in a weak
} resonance at eigenvalue $\lambda^{*}$ at $t=0$.
Write $\lambda_{1}(t, \epsilon), \lambda_{2}(t, \epsilon)$ for the eigenvalues of $M(t, \epsilon) . \lambda_{1}$ and $\lambda_{2}$ are continuous functions, but are not generally differentiable at resonances [25]. However, we can work with functions that determine the eigenvalues that are smooth. Define [9]

$$
\begin{aligned}
& \mu(t, \epsilon)=\left(\lambda_{1}(t, \epsilon)-\lambda_{2}(t, \epsilon)\right)^{2} / 4 \\
& \lambda(t, \epsilon)=\left(\lambda_{1}(t, \epsilon)+\lambda_{2}(t, \epsilon)\right) / 2 .
\end{aligned}
$$

$\lambda$ and $\mu$ may be expressed in terms of $M(t, \epsilon)$ by

$$
\begin{aligned}
\lambda(t, \epsilon) & =\operatorname{Trace}\{M(t, \epsilon) / 2\} \\
\mu(t, \epsilon) & =(\operatorname{Trace}\{M(t, \epsilon) / 2\})^{2}-\operatorname{Det}\{M(t, \epsilon)\} \\
& =\frac{1}{4}(a-d)^{2}+b c .
\end{aligned}
$$

The smoothness of $\lambda$ and $\mu$ and their independence from coordinates follows from (10) and (11) and the smoothness of $M$.

The condition for coincident and resonant eigenvalues is $\mu=$ 0 . Indeed, the condition for strong resonance of $M$ is $\mu=0$ and $b$ and $c$ not both zero and the condition for weak resonance of $M$ is $\mu=0$ and $b=c=0$.

The assumption of weak resonance at $(t, \epsilon)=(0,0)$ implies that

$$
M(0,0)=\left(\begin{array}{cc}
\lambda(0,0) & 0 \\
0 & \lambda(0,0)
\end{array}\right) .
$$

It follows from (13) that

$$
\mu(0,0)=0 .
$$

Starting from (12), we compute some derivatives of $\mu$ and evaluate them at $(t, \epsilon)=(0,0)$. The derivative of $\mu$ with respect to $t$ evaluated at $(0,0)$ is written as $\left.\mu_{t}\right|_{0}$

$$
\begin{aligned}
\mu_{t} & =(a-d)\left(a_{t}-d_{t}\right) / 2+b_{t} c+b c_{t} \\
\mu_{\epsilon} & =(a-d)\left(a_{\epsilon}-d_{\epsilon}\right) / 2+b_{\epsilon} c+b c_{\epsilon} \\
\left.\mu_{t}\right|_{0} & =0 \\
\left.\mu_{\epsilon}\right|_{0} & =0 \\
2 A & =\left.\mu_{t t}\right|_{0}=\left.\left[\left(a_{t}-d_{t}\right)^{2} / 2+2 b_{t} c_{t}\right]\right|_{0} \\
2 B & =\left.\mu_{\epsilon \epsilon}\right|_{0}=\left.\left[\left(a_{\epsilon}-d_{\epsilon}\right)^{2} / 2+2 b_{\epsilon} c_{\epsilon}\right]\right|_{0} \\
2 H & =\left.\mu_{t \epsilon}\right|_{0}=\left.\left[\left(a_{t}-d_{t}\right)\left(a_{\epsilon}-d_{\epsilon}\right) / 2+b_{t} c_{\epsilon}+b_{\epsilon} c_{t}\right]\right|_{0} .
\end{aligned}
$$

Then, for small $(t, \epsilon)$, we have the Taylor series

$$
\mu(t, \epsilon)=A t^{2}+2 H t \epsilon+B \epsilon^{2}+\text { h.o.t. }
$$

Approximating to second order in $\epsilon$ and $t$ by neglecting the third-order and higher order terms, we get

$$
\mu(t, \epsilon)=A t^{2}+2 H t \epsilon+B \epsilon^{2} .
$$

All the following results analyze the approximation (16) and thus yield results about (15) and the power system differential equations that are only accurate to second order in $\epsilon$ and $t$.

We make the generic assumption that $A \neq 0$. We claim that the locus of (16) is a parabola in the complex plane. For $\epsilon \neq 0$, it is convenient to define scaled variables

$$
\mu^{\prime}=\frac{\mu}{A \epsilon^{2}}, \quad t^{\prime}=\frac{t}{\epsilon}, \quad H^{\prime}=\frac{H}{A}, \quad B^{\prime}=\frac{B}{A}
$$

so that

$$
\mu^{\prime}=t^{\prime 2}+2 H^{\prime} t^{\prime}+B^{\prime} .
$$


Taking imaginary and real parts of (18) yields

$$
\begin{aligned}
\mu_{i}^{\prime} & =2 H_{i}^{\prime} t^{\prime}+B_{i}^{\prime} \\
\mu_{r}^{\prime} & =t^{\prime 2}+2 H_{r}^{\prime} t^{\prime}+B_{r}^{\prime} .
\end{aligned}
$$

In the case $H_{i}^{\prime} \neq 0$, (19) can be written

$$
t^{\prime}=\frac{\mu_{i}^{\prime}-B_{i}^{\prime}}{2 H_{i}^{\prime}}
$$

and (20) becomes

$$
\mu_{r}^{\prime}=\frac{1}{4 H_{i}^{\prime 2}}\left(\mu_{i}^{\prime}-B_{i}^{\prime}+2 H_{i}^{\prime} H_{r}^{\prime}\right)^{2}+B_{r}^{\prime}-H_{r}^{\prime 2}
$$

Equation (22) is a parabola with principal axis parallel to the real axis and maximum curvature $1 /\left(4 H_{i}^{\prime 2}\right)$. (16) is simply a rescaling of (22) by $|A| \epsilon^{2}$ that is rotated through $\angle A$. Hence, (16) is a parabola with principal axis parallel to $A$ and maximum curvature $1 /\left(4 H_{i}^{\prime 2}|A| \epsilon^{2}\right)$. The parabola (16) only changes its scale as $\epsilon$ changes; in particular, its scale is proportional to $\epsilon^{2}$.

In the case $H_{i}^{\prime}=0$, (19) becomes

$$
\mu_{i}^{\prime}=B_{i}^{\prime}
$$

and (23) and (20) show that (18) lies in a horizontal line and is a degenerate parabola. Hence, (16) lies in a line in the direction of $A$ and is a degenerate parabola.

For $\epsilon=0$, a weak resonance is encountered at $t=0$ and

$$
\mu(t, 0)=A t^{2}
$$

which is a ray through the origin in the direction of $A$ and a degenerate parabola. Note that the ray (24) is a smooth curve everywhere (its derivative vanishes at the origin) and is a smoothly embedded curve except at the origin. (16) is a continuous perturbation of (24) as $\epsilon$ varies near zero and, except at the origin, is a smooth perturbation of (24). Thus, for sufficiently small $\epsilon$, (16) is a parabola close to (24) and, except near the origin, is $C^{1}$ close to the ray (24).

We can analyze the relative movement between the eigenvalues $\lambda_{1}-\lambda_{2}$ using (16). Recall that

$$
\lambda_{1}-\lambda_{2}=2 \sqrt{\mu}
$$

For $|t|$ large with respect to $\epsilon, \mu \approx A t^{2}$ and

$$
\lambda_{1}-\lambda_{2} \approx \pm 2 \sqrt{A} t
$$

so that the eigenvalues approach each other or separate approximately along a straight line in the direction $\sqrt{A}$.

The line through the origin parallel to the principal axis of the parabola is given by

$$
\mu=A k \epsilon^{2}, \quad k \in \mathbb{R} .
$$

The intersection between the parabola (16) and the line (27) satisfies

$$
A t^{2}+2 H t \epsilon+B \epsilon^{2}=A k \epsilon^{2} \text {. }
$$

Dividing (28) by $A \epsilon^{2}$ and taking the imaginary part yields, at the intersection

$$
t=-\frac{B_{i}^{\prime} \epsilon}{2 H_{i}^{\prime}} \quad \text { and } \quad k=\frac{B_{i}^{\prime 2}}{4 H_{i}^{\prime 2}}-\frac{H_{r}^{\prime} B_{i}^{\prime}}{H_{i}^{\prime}}+B_{r}^{\prime}
$$

If $k>0$, then 0 is outside the parabola and the perturbation is type $\mathrm{A}$ and if $k<0$, then 0 is inside the parabola and the perturbation is type B. For elaboration of this condition in terms of the position of strong resonances when $t$ is considered to be complex, see [11].

The eigenvalue separation at the intersection, using (25) and (27), is

$$
\lambda_{1}-\lambda_{2}= \pm 2 \sqrt{A} \sqrt{k} \epsilon
$$

The magnitude of the eigenvalue separation at the intersection is proportional to $\epsilon$. According to (30) the sign of $k$ also determines the relative direction of the eigenvalues at the intersection. In type $\mathrm{A}, k>0$ and the eigenvalue relative direction at the intersection is along the same direction (26) as the eigenvalues initially approximately approach or finally leave. In type $\mathrm{B}, k<0$ and the eigenvalue relative direction at the intersection is in the direction $j \sqrt{A}$, which is at $90^{\circ}$ to the direction (26) that the eigenvalues initially approximately approach or finally leave.

In [10], a similar analysis is conducted, but with $t$ considered to be a complex parameter (the power system differential equations are assumed analytic in $t$ so that $\mu(t, \epsilon)$ is analytic in $t)$. The second-order Taylor expansion (16) remains valid and factors into linear terms

$$
\begin{aligned}
\mu(t, \epsilon)=A^{-1}(A t+(H & \left.\left.+\sqrt{H^{2}-A B}\right) \epsilon\right) \\
& \times\left(A t+\left(H-\sqrt{H^{2}-A B}\right) \epsilon\right) .
\end{aligned}
$$

Thus, to second order, the complex roots $t$ of $\mu(t, \epsilon)=0$ lie on two straight lines passing through the origin as $\epsilon$ is varied near zero. This implies that the double root at $(t, \epsilon)=(0,0)$ corresponding to a weak resonance typically perturbs to two strong resonances in the complex plane near zero as $\epsilon$ changes from zero. When the parameterization is restricted to real values of $t$, [10] concludes that, since the two strong resonances generally lie in the complex plane off the real axis, the system generally does not encounter either of the strong resonances as $t$ varies near zero. However, for small $\epsilon$, the proximity of the strong resonances to the origin of the complex plane implies that the system does pass close to the strong resonances as $t$ varies near zero. Thus, the power system passes close to two strong resonances at a general, small perturbation of a weak resonance. 


\section{REFERENCES}

[1] G. Rogers, Power System Oscillations. Norwell, MA: Kluwer, 2000.

[2] Cigré Task Force, "Analysis and Control of Power System Oscillations," Cigré Task Force 07 of Advisory Group 01 of Study Committee 38, Paris, France, Dec. 1996.

[3] IEEE Power Engineering Society, "Inter-Area Oscillations in Power Systems," Systems Oscillations Working Group, IEEE Publication 95 TP 101, Oct. 1994.

[4] D. K. Mugwanya and J. E. Van Ness, "Mode coupling in power systems," IEEE Trans. Power Syst., vol. PWRS-2, no. 2, pp. 264-270, May 1987.

[5] J. E. Van Ness, F. M. Brasch, G. L. Landgren, and S. T. Naumann, "Analytical investigation of dynamic instability occurring at Powerton station," IEEE Trans. Power App. Syst., vol. PAS-99, no. 4, pp. 1386-1395, Jul. 1980.

[6] M. Klein, G. J. Roger, S. Moorty, and P. Kundur, "Analytical investigation of factors influencing power system stabilizer performance," IEEE Trans. Energy Conversion, vol. 7, no. 3, pp. 382-390, Sep. 1992.

[7] H. G. Kwatny and X.-M. Yu, "Energy analysis of load-induced flutter instability in classical models of electric power networks," IEEE Trans. Circuits Syst., vol. 38, no. 12, pp. 1544-1557, Dec. 1989.

[8] H. G. Kwatny and G. E. Piper, "Frequency domain analysis of Hopf bifurcations in electric power networks," IEEE Trans. Circuits Syst., vol. 37, no. 10, pp. 1317-1321, Oct. 1990.

[9] I. Dobson, J. Zhang, S. Greene, H. Engdahl, and P. W. Sauer, "Is strong modal resonance a precursor to power system oscillations?", IEEE Trans. Circuits Syst., pt. 1, vol. 48, no. 3, pp. 340-349, Mar. 2001.

[10] I. Dobson, "Strong resonance effects in normal form analysis and subsynchronous resonance," in Bulk Power System Dynamics and Control V Conf., Onomichi, Japan, Aug. 2001.

[11] V. Auvray, I. Dobson, and L. Wehenkel, "Modifying eigenvalue interactions near weak resonance," in Proc. IEEE Int. Symp. Circuits and Systems, Vancouver, BC, Canada, May 2004

[12] L. Jones, G. Andersson, and V. Knyazkin, "On modal resonance and interarea oscillations in power systems," in Bulk Power System Dynamics and Control V Conf., Onomichi, Japan, Aug. 2001.

[13] B. M. Nomikos and C. D. Vournas, "Modal interaction and PSS design," in Porto Power Tech. Conf., Porto, Portugal, Sep. 2001.

[14] "IEEE Power System Engineering Committee, Eigenanalysis and Frequency Domain Methods for System Dynamic Performance," IEEE Publication 90TH0292-3-PWR, 1989.

[15] N. Kakimoto, A. Nakanishi, and K. Tomiyama, "Instability of interarea oscillation mode by autoparametric resonance," IEEE Trans. Power Syst., vol. 19, no. 4, pp. 1961-1970, Nov. 2004.

[16] A. P. Seyranian, "Sensitivity analysis of multiple eigenvalues," Mechanics of Structures and Machines, vol. 21, no. 2, pp. 261-284, 1993.

[17] C.-M. Lin, V. Vittal, W. Kliemann, and A. A. Fouad, "Investigation of modal interaction and its effects on control performance in stressed power systems using normal forms of vector fields," IEEE Trans. Power Syst., vol. 11, no. 2, pp. 781-787, May 1996
[18] S. K. Starrett and A. A. Fouad, "Nonlinear measures of mode-machine participation," IEEE Trans. Power Syst., vol. 13, no. 2, pp. 389-394, May 1998.

[19] J. Thapar, V. Vittal, W. Kliemann, and A. A. Fouad, "Application of the normal form of vector fields to predict interarea separation in power systems," IEEE Trans. Power Syst., vol. 12, no. 2, pp. 844-850, May 1997.

[20] I. Dobson and E. Barocio, "Scaling of normal form analysis coefficients under coordinate change," IEEE Trans. Power Syst., vol. 29, no. 3, pp. 1438-1444, Aug. 2004.

[21] C. E. J. Bowler, D. N. Ewart, and C. Concordia, "Self excited torsional frequency oscillations with series capacitors," IEEE Trans. Power App. Syst., vol. PAS-92, no. 5, pp. 1688-1695, Sep./Oct. 1973.

[22] IEEE Trans. Power Syst., vol. 7, no. 1, pp. 150-157, Feb. 1992

[23] P. M. Anderson, B. L. Agrawal, and J. E. Van Ness, Subsynchronous Resonance in Power Systems. New York: IEEE Press, 1990.

[24] K. R. Padiyar, Analysis of Subsynchronous Resonance in Power Systems. Norwell, MA: Kluwer, 1998.

[25] T. Kato, Perturbation Theory for Linear Operators, 2nd ed, Berlin, Germany: Springer-Verlag, 1995, ch. 2.1.

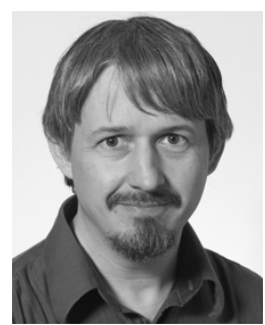

Ian Dobson (SM'98) received the B.A. degree in mathematics from Cambridge, U.K., in 1978 and the $\mathrm{Ph} . \mathrm{D}$. degree in electrical engineering from Cornel University, Ithaca, NY, in 1989.

From 1978 to 1983, he was a Systems Analyst for the British firm EASAMS Ltd. In 1989, he joined the University of Wisconsin-Madison, where he is now a Professor in electrical and computer engineering. His current interests are applications of complex systems and nonlinear dynamics, cascading failure and selforganized criticality in blackout risk, electric power system instabilities, and power electronics.

Emilio Barocio (M'03) received the M.S. degree from the University of Guadalajara, Guadalajara, Mexico, in 1997 and the Ph.D. degree from the Centre for Research and Advanced Studies (CINVESTAV) of the National Polytechnic Institute (IPN) of Mexico in 2003.

From 1997 to 2002, he was a Lecturer in the Department of Mathematics, University of Guadalajara. He was a Visiting Scholar at the University of Wisconsin-Madison in the academic year 2001-2002. Currently, he is an Associate Professor in the Postgraduate Mechanical and Electrical Engineering School, Autonomous University, Nuevo León, Mexico.

Dr. Barocio is a member of the Mexican National Research System. 